\title{
Research on Model of Project Synthetically Management Based on Genetic Algorithm
}

\author{
Xinghua Dang ${ }^{1}$ Lei Wang $^{2}$ \\ ${ }^{1}$ Dean of the school of business management, Xi'an university of technology, Xi'an 710054, P. R. China \\ ${ }^{2}$ school of business management, Xi' an university of technology, Xi' an 710054, P. R. China
}

\begin{abstract}
The multiattribute utility function is used to build a model of project's time, quality and cost synthetically management under resource restriction in this paper, and then the genetic algorithm is introduced to solve the model. Finally, an example and an alternative scheme of synthetically optimizing and managing time, quality and cost of projects in practice are given. The example of XiHe hydropower is presented to show the reasonableness and effectiveness of the proposed method.
\end{abstract}

Key words: Project management, Genetic algorithm, Multiattribute utility Function

\section{Introduction}

Time limit, quality and cost, which are three aims of the construction project management, have dialectic relations with each other, time, quality and cost should be optimized synthetically and planed overall. For the restriction of fund, human resource, technology and geography environment etc, under such limited resource condition the problem of how to get the equilibrium of the three aims is always concerned by the proprietor, contractor and monitor. A great many academicians have been doing some valid researches on the optimization of quality-cost and time-cost. For example, Jia XP etc.(2003) analyzed multi-goal course system optimization problems[1], it utilized hereditary algorithm to realize Pareto optimal collection answer, and utilize TOPSIS method to select the Pareto coordinating answer; Wang J(2004) discussed how to utilize multiattribute utility function to establish the model of synthetically optimization of project's time, quality and cost in theory[2]; Zhang LY(2004) utilized hereditary algorithm and MATLAB method to realize the balanced distribution of project's resource, this method overcome the deficiency of the traditional resource balance method[3]; Luo G(2004) analyzed the hereditary algorithm application in the network plan resource optimization, which was used to solve two kinds of optimization problems under restrained resource condition [4]; Guo Y(2005) utilized hereditary algorithm to study the multi-project resource balance problem[5]; Mirelle(2002) studied the proportionate relationship of every item in quality-cost, such as confirming the proportion of prevent cost and appraise cost to make the total cost minimum [6]; Babu(1996) thought that accelerating the speed of the project would influence the quality. The author established linear programming model to research the balanced relationship of time, quality and cost [7]; Khang (1999) utilized the linear programming model which established in Babu (1996) to study cement plant's time, quality and cost synthetically optimization and management [8].

Through synthesizing the current research situation we can find that the present research concentrate on two aspects, one is the application of hereditary algorithm in construction project management; the other is the optimization of time-cost and quality-cost. But nowadays, there are just a few models to solve the optimization question of time and quality. The study on synthetically optimization question of time, quality and cost especially on the project synthetically optimization question under resource restriction are still blank in a paper or form. Under the condition of predecessor's research achievements, this article used multiattribute utility function to establish the project's time, quality and cost optimization. And it introduced the genetic algorithm to solve the model. This article provided some theory evidence and reality reference value for construction project's synthetically management.

\section{Multiattribute utility function of time, quality and cost}

\subsection{The definition of the multiattribute utility function}


The utility theory stemmed from J.Benthan (1948) initially was the tool of favor comparison among individuals. Afterwards, it was expanded in the study of the demand theory of consumption and economics, privation and public policy's management and control. With regard to the question of multi-target's tackle, the multiattribute utility function can sort the un-inferior objective collection in principle and obtain the highest utility's un-inferior explanation. The work of the object management is to solve the question of multi-target's tackle for choosing the shortest time, optimized quality, the lowest cost act as the target of control [2].

Among the synthetically optimization and domination of the time, quality and cost, this article used the method of avail analysis to standardize it. We choose $U(T, Q, C)$ act as multiattribute function, it can express as [9]:

$$
\mathrm{U}: \quad(\mathrm{T}, \mathrm{Q}, \mathrm{C}) \Rightarrow U(T, Q, C) \in U \in R
$$

Among them: R represents real number collection. Assumed that the time limit for a project (T), quality (Q) and cost (C) as attribute variables for the function, its preference relationship satisfied with:

$\left(T^{\prime}, Q^{\prime}, C^{\prime}\right) \succ\left(T^{\prime \prime}, Q^{\prime \prime}, C^{\prime \prime}\right) \Leftrightarrow U\left(T^{\prime}, Q^{\prime}, C^{\prime}\right) \stackrel{>}{=} U\left(T^{\prime \prime}, Q^{\prime \prime}, C^{\prime \prime}\right)$ It also can be thought as the function with largest valid during the process of the synthetically optimization and control towards these three aims.

\subsection{Decompose of the multiattribute utility function}

In practice, the optimization and control of these three varies of the multiattribute utility function were very difficult. First, the article analyzed the relation of each two varies, then synthesized these double varies avail function and analyzed synthetically three varies.

According to the resolve theorem of the multiattribute utility function, $\mathrm{U}(\mathrm{T}, \mathrm{Q}, \mathrm{C})$ can adopt the style of multiplication and division.

$$
U(T, Q, C)=U(C, Q) \bullet U(T, C) / U(Q, T)
$$

$\mathrm{U}(\mathrm{C}, \mathrm{Q})$ represents quality-cost function; $\mathrm{U}(\mathrm{T}, \mathrm{C})$ represents time- cost function; $U(Q, T)$ represents time-quality function.

\subsection{The establishment of the multiattribute utility function}

Because of the multiattribute utility Function $U(T, Q$, C) can divide into utility function $\mathrm{U}(\mathrm{C}, \mathrm{Q}), \mathrm{U}(\mathrm{T}, \mathrm{C})$ and $\mathrm{U}(\mathrm{Q}, \mathrm{T})$, this text used the analytic structure method to establish above-mentioned three pieces of utility function, then obtained the multiattribute utility function through the theorem of resolving of multiattribute utility function.

The cost of every good product

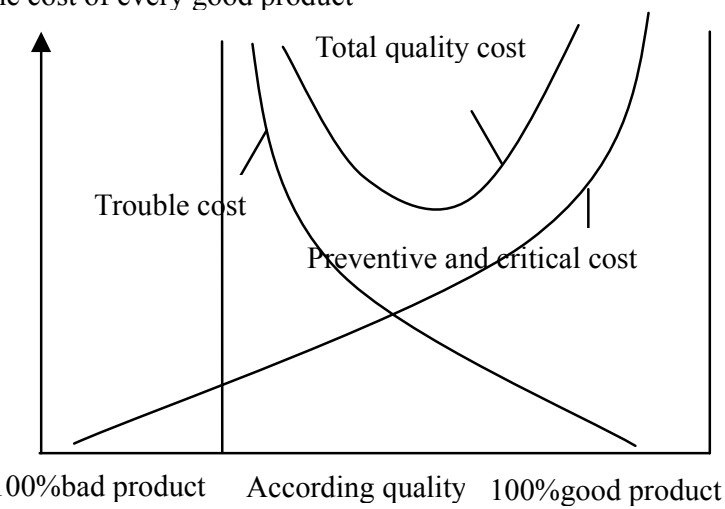

\section{Model Establishment}

\subsection{Quality-cost relationship models}

The optimization question of the quality cost is the coordination question of the systematic dependability and economy. The project is complicated network system engineering, the theory of optimization of the network system can be used to configure the relation between quality and cost rationally and then realize the rational distribution of the quality cost.

In project construction, often confirm first that a quality goal is the systematic reliability, then carry on the controlling optimally of cost on the premise of this quality goal and make the project cost rational to be lowest. In a situation that the reliability of the system is limited the model that resolves the optimum expenses of system is:

$$
\begin{aligned}
& C=\min \sum_{i=1}^{n} f_{i}(R(i)) \\
& \text { s.t. } R=\xi(R(1), R(2), \cdots, R(n)) \leq R_{S} \\
& 0 \leq R(i) \leq 1, i=1,2, \cdots, n
\end{aligned}
$$

Among them: $\xi(R(1), R(2), \cdots, R(n)) \quad$ is total reliability degree of the system when $R(1), R(2), \cdots, R(n)$ are the reliability degree of the subsystem, $R_{\mathrm{s}}$ is the limit value of the systematic reliability degree.

Quality-cost includes: prevent cost, appraise cost, 
internal trouble cost and external trouble cost. Among them, prevent cost and appraise cost are named preventive and critical cost. The famous quality management expert of Dr. M. Juran point out that [10]: the favorable ratio of these four parts is $10 \%, 40 \%$, $50 \%$. The favorable ratio of the quality-cost can be indicated by using "optimum quality cost model" as shown in Figure 1.

Fig. 1: Quality-Cost Relation Model.

According to the quality-cost model, this article uses secant function to simulate preventive and critical cost and uses cotangent function to simulate trouble cost and finally succeed in getting the quality-cost relationship model as:

$U(C, Q)=\min \sum C_{i}^{N}\left\{\begin{array}{l}\omega_{1}[\tan (\pi / 2) \bullet R(i)]^{p_{1}} \\ +\omega_{2}[c \tan (\pi / 2) \bullet R(i)]^{p_{2}}\end{array}\right\}$

Among them: $C_{i}^{N}$ represents the common direct expenses of working procedure i, $\omega^{1}$ and $\omega^{2}$ each represents the proportion of the preventive and critical cost to the total quality-cost and that of the trouble cost to the total quality-cost. In this case, $\omega^{1}=\omega^{2}=0.5$, R (i) represents the reliable extent of the secondary system, P1, P2 represent the cost-increasing index. The value of P1 and P2 depends on distinct corporation for its level of technology and management distinguishes.

\subsection{Time-cost relationship models}

The cost refers to the expenses that are expended when the corresponding measure need to take to realize the goal of time limit for a project or contract in time limit for a project. The optimization of cost means that under the prerequisite of meeting contract time limit for a project in time limit for a project the rational duration of every process has to be fixed and the project cost has to be made lowest rationally. To reduce calculation, suppose that the relation of time and cost are linearity and time delay must cause cost's increase. Both are in inverse ratio. So the relation of time limit and cost can be denoted by figure 2 .

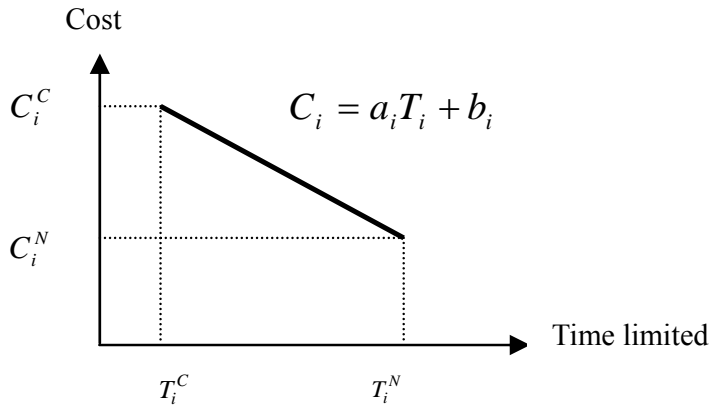

Fig. 2: the Relationship between Time and Cost of Working Procedure i.

Among them: The time-cost slope is $a_{i}=\frac{C_{i}^{N}-C_{i}^{C}}{T_{i}^{N}-T_{i}^{C}}$

The intercept is

$b_{i}=\frac{C_{i}^{C} T_{i}^{N}-C_{i}^{N} T_{i}^{C}}{T_{i}^{N}-T_{i}^{C}}$

The aim of the time cost optimum is to satisfy with the request of the time through the compression of key point of the way's time and at the same time make the expenditure of the project minimum and thus succeed in getting the time-cost relationship model as follows:

$$
\begin{aligned}
& U(T, C)=\min \sum C_{i}=\min \sum\left(a_{i} T_{i}+b_{i}\right) \\
& =\min \sum\left\{\begin{array}{l}
{\left[\left(C_{i}^{N}-C_{i}^{c}\right) \bullet T_{i} /\left(T_{i}^{N}-T_{i}^{C}\right)\right]} \\
+\left[\left(C_{i}^{C} T_{i}^{N}-C_{i}^{N} T_{i}^{C}\right) /\left(T_{i}^{N}-T_{i}^{C}\right)\right]
\end{array}\right\}
\end{aligned}
$$

Here, $T_{i}^{N}$ Represents fulfill the working procedure's common abidance time; $T_{i}^{C}$ represents fulfill the working procedure's limit time, $C_{i}^{C}$ represents the working procedure limit expenditure of $i$, $C_{i}^{N}$ represents the working procedure common direct expenditure of $i, T^{i}$ represents the procedure practical abidance time of i. With $T_{i}^{N} \geq T_{i}^{C}>0, \quad C_{i}^{C} \geq C_{i}^{N}>0$.

\subsection{Times-quality model}

Optimization of quality means that under meeting plan time limit for a project or the prerequisite of time limit for a project of contract in time limit for a project the 
rational credit rating (reliability) of every process has to be confirmed and the systematic reliability has to be made largest. To reduce calculation, we also can suppose that time and quality are linearity (the figure is omitted), among it, the slope of time limit and quality relationship line is $d_{i}=\frac{Q_{i}^{N}-Q_{i}^{C}}{T_{i}^{N}-T_{i}^{C}}$. The shorter of abidance time, the lower of reality quality. Both are in direct ratio, with $\mathrm{Q}=0$, when $\mathrm{T}=0$.

The question of the time limit and quality optimization means that the time limit has to be shortest under the condition of the definite quality or the quality has to be best under the condition of the definite time limit. To improve the reliability degree of the project quality as much as possible, the time limit and quality relationship model under the normal plan is:

$$
\begin{aligned}
& U(Q, T)=\max \sum Q_{i}=\max \sum d_{i} T_{I} \\
& =\max \sum\left\{\left(Q_{i}^{N}-Q_{i}^{c}\right) \bullet T_{i} /\left(T_{i}^{N}-T_{i}^{C}\right)\right\}
\end{aligned}
$$

Here, $Q_{i}^{N}$ represents the normal quality of the procedure i, $Q_{i}^{C}$ represents the high-point quality of the procedure $\mathrm{i}$, and $Q_{i}^{N} \geq Q_{i}^{C}>0, T_{i}^{N} \geq T_{i}^{C}>0, \sum T_{i}=\mathrm{T}$, the meanings of other signals are same as above.

\subsection{Establishment of time, quality}

\section{and cost synthetically}

\section{management model}

Through the above analysis, the synthetically optimization of the time, quality, cost is to make the multiattribute utility function get its avail's max. That is to say its short-cut process minimum. It also means that namely $\mathrm{U}(\mathrm{C}, \mathrm{Q}), \mathrm{U}(\mathrm{T}, \mathrm{C})$ both get its min; $\mathrm{U}(\mathrm{Q}$, $\mathrm{T})$ gets its max. Thereby, we can build a model of project's time quality and cost synthetically optimization and control model as follows:

$$
U(T, Q, C)=\min [U(C, Q) \bullet U(T, C) / U(Q, T)]
$$

$$
\begin{aligned}
& \left\{U(C, Q)=\min \sum C_{i}^{N}\left\{\begin{array}{l}
\omega_{1}[\tan (\pi / 2) \bullet R(i)]^{p_{1}} \\
+\omega_{2}[c \tan (\pi / 2) \bullet R(i)]^{p_{2}}
\end{array}\right\}\right. \\
& U(T, C)=\min \sum C_{i}=\min \sum\left(a_{i} T_{i}+b_{i}\right) \\
& \text { st. }\left\{=\min \sum\left\{\begin{array}{l}
{\left[\left(C_{i}^{N}-C_{i}^{c}\right) \bullet T_{i} /\left(T_{i}^{N}-T_{i}^{c}\right)\right]} \\
+\left[\left(C_{i}^{C} T_{i}^{N}-C_{i}^{N} T_{i}^{C}\right) /\left(T_{i}^{N}-T_{i}^{C}\right)\right]
\end{array}\right\}\right. \\
& U(Q, T)=\max \sum Q_{i}=\max \sum d_{i} T_{I} \\
& =\max \sum\left\{\left(Q_{i}^{N}-Q_{i}^{c}\right) \bullet T_{i} /\left(T_{i}^{N}-T_{i}^{C}\right)\right\} \\
& R=\xi(R(1), R(2), \cdots, R(n)) \leq R_{S}, 0 \leq R(k) \leq 1, \\
& k=1,2, \cdots, n, \omega_{1}=\omega_{2}=0.5
\end{aligned}
$$

\section{Genetic algorithm design and model solution}

Considering the characteristic of the engineering project, this article makes use of the genetic algorithm to obtain the solution of the model of synthetically optimization and control of the time, quality-cost. Genetic algorithm is an accidental indexical algorithm, which use the nature select and nature genetic mechanism for reference. This method was raised by the U.S. professor J. Holland. Its main peculiarity is group indexical tactics and individual information exchange in group and it doesn't depend on grads information in the index. [11]

Selection, crossover and mutation are three-control factor of genetic algorithm, those who constitute the genetic operation. Parameter coding, the enactment of initial group, the estimate of mode rational function, the design of genetic operation and the enactment of control figure constitute the core content of genetic algorithm. [12]

\subsection{The general method of genetic optimization}

(1)Confirm the group dimension $\mathrm{n}$ and use the accidental method or any other method to produce $n$ possible key. $X^{i}(\mathrm{k})(1 \leq \mathrm{i} \leq \mathrm{n})$ to constitute the initial solution group.

(2)To each individual, calculate its suit degree.

(3)To each individual, calculate its existence probability. Then, design an accidental select organ, 
according to $P^{i}(\mathrm{k})$ produce breeding individual through accidental method.

(4)Bring about next generation solution group. Choose two breeding individual $X_{1}(\mathrm{k}), X_{2}(\mathrm{k})$, integrate $X_{1}(\mathrm{k}), X_{2}(\mathrm{k})$ into two other new individual $X_{1}(\mathrm{k}+1), X_{2}(\mathrm{k}+2)$ according to certain combination regulation until the new generational individual shaped over.

(5)Repeat the step (2)-(4) until satisfy the condition of the procedure's end.

\subsection{The algorithm realization}

(1) Parameter coding. The cost optimum question belongs to the numerical value optimum question. To facilitate making contact with traditional algorithm, select floating decimal number coding. Let each corresponding floating decimal number of each task's cost and dependable degree to be a gene and time gene correspond with working procedure lasting time chromosome and cost gene correspond with cost chromosome and dependable degree gene correspond with quality chromosome and each chromosome correspond with a solution of the question.

(2) Adapt degree function. One important character of using genetic algorithmic is that it can take the target function value as the search information. The target of this text is to seek the target function min. The target function can be taken as adapt degree target directly but need to switch adapt degree. Base on the experience of actual applying, the linearity degree switch of adapt degree should be selected.

(3) Genetic factor. Genetic factor includes selection factor, cross over factor and mutation factor. To speed up the constringency speed of genetic algorithm and increase the diversity of group while retaining suitability some lower individual in the choosing operation we adopt developmental crossover factor and developmental mutation faction. The concrete as follows:

$$
p_{c}=p_{c 0}\left(\frac{T-t}{T}\right), p_{m}=p_{m 0}\left(\frac{T-t}{T}\right)
$$

$p_{c}, p_{m}$ represent the across and aberrance ratio evolved until now; $p_{c 0}, p_{m 0}$ represent the initial across and aberrance ratio; $t$ represents current evolve number; $\mathrm{T}$ represents the enacted max evolve number.

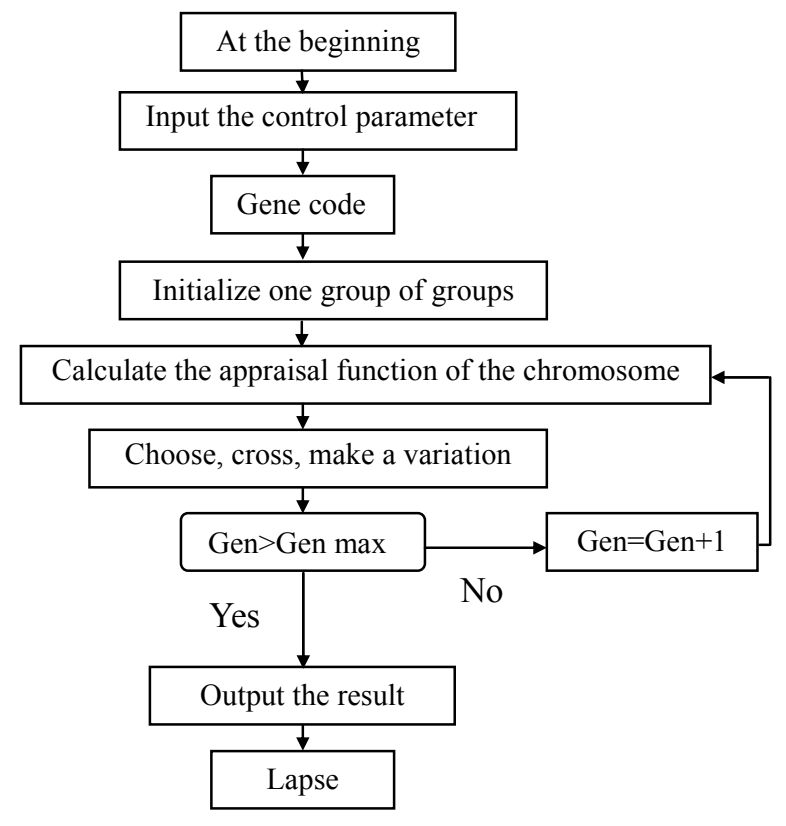

Fig. 3: the Process of Genetic Arithmetic

(4) System parameter and occur repeatedly ending condition. Set the length of coding bunch as the total number of web planned procedure, the scale of colony is $M$ and the scale of common $M$ is $20 \sim 100$; the initial across probability is $p_{c 0}$ and its common scale is $0.4 \sim 0.99$; the initial aberrance probability is $p_{m 0}$ and its common scale is $0.0001 \sim 0.1$; the ending algebra $\mathrm{T}$ is a parameter to denote the ending condition of descendible arithmetic if the descendible arithmetic doesn't stop until it run to the pointed evolve number and take the best individual of the currently colony as the best key of the requested question. [13]Furthermore, if the descendible arithmetic has Run $\mathrm{n}$ times continually ( $n$ can be selected based on the actual situation), the square difference of adapt degree is smaller than a very small value then the descendible arithmetic can be stopped ahead. The flow of descendible arithmetic is denoted by figure 3 .

\section{Example of application}

This example is taken from one hydropower station project. This project lies in XiHe where the mainstream of HanJiang River. It is the third hydropower station of seven steps of planning of the upper reaches of HanJiang River (the Section of Shaanxi province), it is a medium-sized hydroelectric project mainly used for generating electricity. The dam is 60.8 meters high and the roof of the dam is 352 meters long. The installed capacity of the reservoir is 229 million cubic meters 
and the regulate capacity of reservoir is 20 million cubic meters. Project installation is $3 \times 60,000$ kilowatts and annual average generation is 494 million kilowatt hours. The annual generation is $4.94 \times 108 \mathrm{kWh}$. It is utilized 2710 hours every year. It is the key irrigation works of Shaanxi Province for building the HanJiang River green economic long corridor. Total installed capacity is 180,000 kilowatts. The project simplicity web planning is already given in figure 4 . The shortest lasting time of each working procedure, common lasting time, longest lasting time, project direct expense's ratio are given in table 1. Indirect expense ratio is 70,000 Yuan per day. According to the common lasting time the indirect fee to finish the plan is $75,600,000$ Yuan. The contract time of the project is 3 year. The planning achieve quality degree is department excellent project.

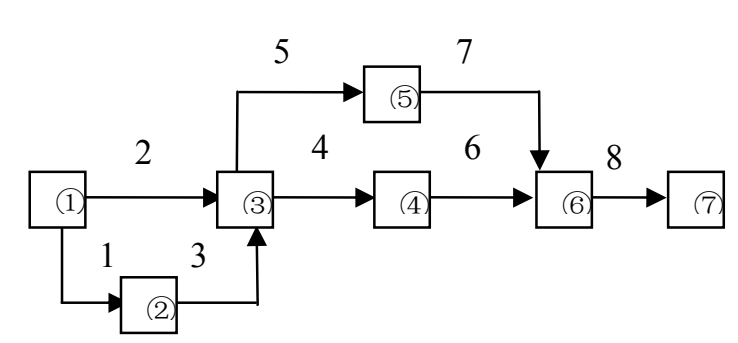

Fig. 4: the Concise Web of XiHe Hydropower station

Annotate: the array head in this figure are the code name of that procedure, its true meaning as following: 1 -cofferdam, 2-left lead flow dam, 3-right lead flow dam, 4-lead flow dyke, 5-cut flow, 6-sluice, 7-build dam, 8-machine install and debug.

\begin{tabular}{|c|c|c|c|c|c|c|c|}
\hline $\begin{array}{c}\text { Procedure } \\
\text { code } \\
\text { name }\end{array}$ & $\mathrm{i}$ & $\mathrm{j}$ & $\begin{array}{c}\text { Min } \\
\text { duration } \\
\text { (day) }\end{array}$ & $\begin{array}{c}\text { Normal } \\
\text { duration } \\
\text { (day) }\end{array}$ & $\begin{array}{c}\text { Max } \\
\text { Duration } \\
\text { (day) }\end{array}$ & $\begin{array}{c}\text { Direct cost } \\
\text { (ten thousands Yuan } \\
\text { per day RMB) }\end{array}$ & $\begin{array}{c}\text { Direct cost ratio } \\
\text { (ten thousands Yuan } \\
\text { per day RMB) }\end{array}$ \\
\hline 1 & 1 & 2 & 45 & 90 & 135 & 225 & 25 \\
\hline 2 & 1 & 3 & 180 & 300 & 360 & 1200 & 40 \\
\hline 3 & 2 & 3 & 150 & 270 & 330 & 891 & 33 \\
\hline 4 & 3 & 4 & 60 & 75 & 105 & 90 & 12 \\
\hline 5 & 3 & 5 & 60 & 90 & 120 & 297 & 33 \\
\hline 6 & 4 & 6 & 300 & 450 & 570 & 1800 & 60 \\
\hline 7 & 5 & 6 & 30 & 45 & 60 & 270 & 50 \\
\hline 8 & 6 & 7 & 30 & 60 & 90 & 300 & \\
\hline
\end{tabular}

Table 1 Duration, Direct cost and Direct Cost Ratio

Under the resource restriction condition in order to arrange each working procedure's lasting time and quality degree to make project cost reasonable min and to achieve of target quality degree require we use the model as above to analyze optimized.

Import each parameter in web planning to the procedures, and set each parameter of genetic algorithm as follows: $p_{c 0}=0.6, p_{m 0}=0.01, \mathrm{~T}=150$, group dimension $\mathrm{M}=100, \omega_{1}=\omega_{2}=0.5$. Let the quality target to be the main target. Planning time is 1080 days; the optimization result through running the procedure is given in table 2. At the same time, we obtain the calculate time through project optimization is 856.5days. The corresponding system total cost is 527670000 Yuan and the achievable quality degree is 0.83 , which satisfy the requirement of the project target quality degree. These optimization numbers can also be used to control cost and bring the schedule control, and cost control, quality control together.

\begin{tabular}{|c|c|c|c|c|c|c|c|c|}
\hline Procedure & $1 \sim 2$ & $1 \sim 3$ & $2 \sim 3$ & $3 \sim 4$ & $3 \sim 5$ & $4 \sim 6$ & $5 \sim 6$ & $6 \sim 7$ \\
\hline Duration (day) & 75 & 270 & 255 & 66 & 72 & 390 & 37.5 & 46.5 \\
\hline $\begin{array}{c}\text { cost (ten thousands } \\
\text { Yuan RMB) }\end{array}$ & 2400 & 12690 & 10200 & 1254 & 2880 & 18330 & 2362.5 & 2650.5 \\
\hline Reliability degree & 0.80 & 0.8 & 0.87 & 0.82 & 0.81 & 0.82 & 0.84 & 0.9 \\
\hline
\end{tabular}

Table 2: Result of Parameter Optimize 


\section{Conclusions}

This article makes time, quality and cost management to be the foundation of project synthetically management. It build a multi-target optimization and control model through the genetic algorithm and obtain the parameter optimization result which based on the ensured quality degree and appropriate times each working procedure's minimum cost under resource restriction. It provides a brand-new perspective for nowadays construction project's synthetically management; it also has certain theory evidence and reality reference value.

\section{Acknowledgement}

This work is partially supported by Soft Science Foundation of Shaanxi Province, China (No. 2006KR32).

\section{References}

[1] X.P. Jia, Multi-objective Optimization for Process System by Means of Genetic Algorithms. Journal of Qingdao University of Science and Technology, 2: 33-36, 2003.

[2] J. Wang, E. L. Liu and G. Luo, Analysis of time-cost-quality tradeoff optimization in construction project management, Journal of Systems Engineering, 19: 148-153, 2004.

[3] L.Y. Zhang and J. P. Zhang, Genetic Algorithms Based on MATLAB of Construction Project Resource Leveling, Journal of Industrial Engineering and Engineering Management, 1:52-55, 2004.

[4] G. Luo and E.L. Liu, Resource Planning Optimization in Network Schedule Using Genetic Algorithms, Journal of Tianjin University, 37:179-183, 2004.

[5] Y. Guo and X. X. Ning, Using Genetic Algorithms for Multi-project Resource Balance, Systems Engineering-theory \& Practice, 10: 78-82, 2005.

[6] M.G. Battika and Qualicon, Counter-Based System for Construction Quality Management, Journal of Construction Engineering and Management, 128:164-173, 2002.

[7] A.J.G. Babu and N. Suresh, Project management with time, cost, and quality considerations, European Journal of Operational Research, 88: 320-327, 1996.

[8] B. Khang and Y.M. Myint, Time, cost and quality trade-off in project management: A case study.
International Journal of Project Management, 17: 249-256, 1999.

[9] Operational Research writes group, editor. Operation Research. Beijing: Qinghua University Press, pp.256-257, 1990.

[10]J.M. Juran, editor. Quality Control Manual. Shanghai: shanghai technology publishing company, pp.223-225, 1997.

[11] G. L. Chen and X. Wang, editor. Descendible arithmetic and its applying. Beijing: people mail publishing company, pp.166-167, 1996.

[12] Tarek Hegazy, and Nagib Wassf, Cost Optimization in Project with Repetitive Nonserial Activities. Journal of Construction Engineering and Management, 128:181-191, 2001.

[13] S.S. Lea, C. H. Yang and J. C. Huang, Resource Leveling in Construction by Genetic Algorithm-Based Optimization and its Decision Support System Application. Automation in Construction, 10: 27-41, 2000. 